\title{
Effect of Combined Training on Muscle Co-Activation and Functional Capacity in Older Women: a Pilot Study
}

\section{Efeito do Treinamento Combinado na Coativação Muscular e Capacidade Funcional em Mulheres Idosas: um Estudo Piloto}

\author{
Guilherme da Silva Rodrigues ${ }^{\mathrm{a}}$; Vagner Ramon Rodrigues Silva ${ }^{\mathrm{b}}$; Bruno Luiz de Souza Bedo ${ }^{\mathrm{a}}$; Mariana Luciano de \\ Almeida ${ }^{c}$; Andressa Crystine da Silva Sobrinho ; André Katayama Yamadad; Carlos Roberto Bueno Júnior ${ }^{\mathrm{d}}$
}

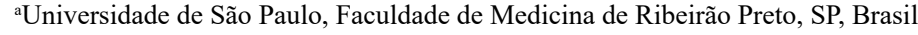

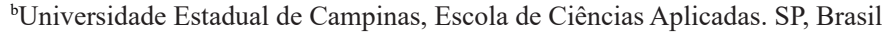

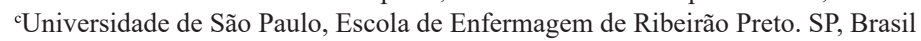 \\ ¿Universidade de São Paulo, Escola de Educação Física e Esporte de Ribeirão Preto. SP, Brasil. \\ *E-mail: buenojr@usp.br
}

\begin{abstract}
Aging is characterized by morphological and functional declines, including impairment in muscle performance and functional capacity. Herein, changes were investigated in strength promoted by combined training and its effects on muscle co-activation in older women. After tree-week adaptation period, thirteen healthy older women (60.2 \pm 6.2 years) underwent a 12-week training program, three sessions a week, one hour per session, divided into 30 minutes of aerobic exercise on a treadmill or cycle ergometer and 30 minutes of strength training. Muscle strength tests and cardiopulmonary fitness assessment were performed before and after the intervention. The results herein showed improvements in strength, functional capacity, and lower limb muscle activation, but no differences in muscle co-activation. In conclusion, the data of this study suggest that 12 weeks of combined training exercise may not be effective in inducing muscle co-activation but may help prevent or mitigate the decline in muscle performance and functional capacity in the older population.
\end{abstract}

Keywords: Electromyography. Aging. Exercise. Health Evaluation.

\section{Resumo}

O envelhecimento é caracterizado por declínios morfológicos e funcionais, incluindo prejuízo no desempenho muscular e capacidade funcional. Aqui, investigamos as mudanças na força promovidas pelo treinamento combinado e seus efeitos na coativação muscular em mulheres idosas. Após três semanas de adaptação, treze idosas saudáveis (60,2 $\pm 6,2$ anos) realizaram um programa de treinamento por 12 semanas, três sessões semanais, uma hora por sessão, divididas em 30 minutos de exercício aeróbio em esteira ou cicloergômetro e 30 minutos de treinamento de força. Testes de força muscular e avaliação da aptidão cardiopulmonar foram realizados antes e após à intervenção. Nossos resultados mostraram melhorias na força, capacidade funcional e ativação muscular dos membros inferiores, mas nenhuma diferença na coativação muscular. Em conclusão, nossos dados sugerem que 12 semanas de exercícios de treinamento combinado podem não ser eficazes na indução da coativação muscular, mas podem ajudar a prevenir ou diminuir o declínio muscular e a melhora da capacidade funcional na população idosa.

Palavras-chave: Eletromiografia. Envelhecimento. Exercício Físico. Avaliação em Saúde.

\section{Introduction}

The number of individuals aged 60 or over will increase dramatically in the next three decades, with the world population over 60 years of age reaching two billion in 2050, according to the World Health Organization (WHO) ${ }^{1}$. However, aging represents a primary risk factor for chronic diseases, including cardiovascular and neurodegenerative conditions ${ }^{2}$. Aging process is characterized by functional and morphological declines, such as the reduction in strength, muscle mass, and cardiorespiratory capacity. Although this decline occurs in both men and women, it is more evident in women after menopause ${ }^{3}$.

Longitudinal evidence demonstrates that in individuals aged 75 years, muscle mass is lost at a rate of $0.64-0.7 \%$ per year in women and $0.8-0.98 \%$ per year in men $^{4}$. Function is lost more rapidly, and evidence demonstrates that at the age of 75 , strength is lost at a rate of $3-4 \%$ per year in men and
$2.5-3 \%$ per year in women ${ }^{4}$. Weakness and wasting have several consequences, such as the inability to independently perform activities of daily living, frailty, and an increased risk of falling, related to depression/social isolation, physical inactivity, and an increased risk of chronic diseases and allcause mortality ${ }^{5,6}$.

Physical activity, which declines with aging, can also protect against muscle atrophy through muscle strength training. Maintaining physical activity is important because it can influence muscle mass/function and metabolic health. Individuals who exercise present better muscle function, muscle quality, and maintenance of type II fiber size ${ }^{7}$. In line with this, combined training, characterized by the realization of aerobic training and muscle strength in the same training session, has been demonstrated to be efficient in older women, since aerobic fitness and muscle strength are the most important physical capacities for this population group. Combined training is little utilized due to the possibility of 
interference in strength capacity ${ }^{8}$. Researchers ${ }^{9}$ demonstrated that combined training realized twice a week (12 weeks), increased muscle performance and functional capacity in older men and another study reported that concurrent exercise also promoted an increase in lower limb strength ${ }^{8}$.

Combined training can lead to co-activation of antagonistic muscles, highlighting its importance for the older community with the main function of increasing joint stability and human movement coordination; co-activation occurs when the agonists activities are limited and their force production has decreased, since the antagonist is exerting a force to the contrary, providing an increase in joint stability, as mentioned previously ${ }^{10}$.

In this scenario, herein the effects of combined training on muscle co-activation, muscular activation, and changes in functional capacity in older women were evaluated. A literature review was performed which confirmed that muscle co-activation has not previously been investigated in older women undergoing a combined training program.

\section{Material and Methods}

\subsection{Study design and subjects}

Following approval from the ethics committee (CAAE 24579513.4.0000.5407), the participants were selected by the "Physical Education for Aging program" of School and Physical Education and Sport of Ribeirão Preto. For inclusion in the study, the following criteria were used: being physically active with minimum practice of 6 months of physical activities considering the short version IPAQ instrument ${ }^{11}$; medical certificate for liberation of physical activity; and women between 50 - 70 years. Participants with less than $75 \%$ adherence to the exercise training sessions were excluded. Thirty participants were included in the eligibility assessment, of which ten participants were excluded as they did not meet the exclusion criteria and/or declined to participate, so twenty participants were allocated to the training adaptation and protocol. Thereafter, three participants missed the assessments, one participant provided incorrect contact information, and three participants missed $25 \%$ or more of the training sessions. In the end, thirteen participants completed the training program.

\subsection{Training adaptation and evaluation}

After the study selection and explanation, the participants were submitted to three-week combined training familiarization period before they initiated pre-intervention tests. The familiarization protocol consisted of two sets of 10 to 12 repetitions of exercises for the main muscular groups with a duration of 30 minutes and aerobic exercises at $50 \%$ of the heart rate reserve for 30 minutes, totaling 60 minutes of training.

The participants were submitted to four physical tests of muscle strength, two of which were targeted to functional capacity, and an aerobic fitness test, in both the pre- and posttraining moments:

- Maximum dynamic load in lower and upper limbs: in a sitting position, one-sided curl with the dominant arm was performed for maximum dynamic load in upper limb and leg press $45^{\circ}$ for lower limb, maintaining the same absolute load pre- and post-intervention, for comparison of the improvement in muscle stimulus through electromyography in the agonist/ antagonist electrical activity. At the post-intervention moment, five minutes of rest were given before the participant performed the test again, aiming to increase absolute load for the analysis of eventual variation in the muscle strength level ${ }^{12}$.

- Sit-to-Stand test: functional strength and resistance of lower limbs pre- and post-intervention were evaluated. The participants were instructed to perform as many repetitions as possible in 30 seconds $^{13}$.

- Elbow flexion and extension: to evaluate functional strength and resistance of upper limbs pre- and postintervention - the participants were instructed to perform as many repetitions as possible in 30 seconds $^{13}$.

- 6-minute walking test: to evaluate aerobic fitness, consisting of walking the longest distance possible in 6 minutes, delimited by a rectangle $(4.57 \times 18.28 \mathrm{~m})^{13}$.

\subsection{Training protocol}

The training program had a duration of 12 weeks, with one hour per session, divided into 30 minutes of aerobic exercise on a treadmill or cycle ergometer and 30 minutes of resistance exercise in the following exercises: leg curl machine, leg extension, leg press machine, lat pull down front machine, direct unilateral biceps curl, triceps in cross over, and chest press, aiming to activate the main muscle groups - pectoralis, arms, shoulders, back, glutes, and legs. We adopted daily undulated periodization for 12 weeks - on the first day of the week the participants realized 5 to 7 repetitions maximum, on the second 10 to 12 , and on the third 15 to 17 , thus providing different muscle stimulus, and, consequently, varying efforts of resistance, hypertrophy, and strength ${ }^{14}$. The cardiorespiratory training intensities were as follows: $70 \%$ of heart rate reserve on the first day, $60 \%$ on the second day, and $50 \%$ on the third, according to Fleck ${ }^{15}$ and a maximum incremental test ${ }^{16}$.

\subsection{Electromyographic data}

Electromyographic activity was evaluated to obtain muscle activation values in the maximum dynamic load, sitto-stand, and elbow flexion and extension tests. A biological signal conditioner Trigno Wireless (Delsys, Inc., Boston Massachusetts, USA) was used, with 16 channels, 16 bits resolution, and sampling at $2000 \mathrm{~Hz}$. Prior to electrode fixation, trichotomy and skin cleaning were performed by abrasion with fine sandpaper and alcohol (70\%). The electrodes were positioned following the proposed guidelines 
for Surface EMG for Non-Invasive Assessment of Muscles ${ }^{17}$, on the brachial biceps and triceps for upper limbs. In addition, the rectus femoris, vastus medialis and lateralis, femoris biceps, and semitendinosus were used for lower limbs.

To provide normalized data during the test execution, isometric maximum voluntary contractions (MVC) lasting 10 seconds were performed in the same exercises of maximum dynamic load in lower and upper limbs ${ }^{18}$. All the electromyographic data were smoothed by a Butterworth filter of fourth order with cut-off frequency of 20-500 Hz (band pass) and the root mean square (RMS) was calculated. Data analyses were performed using programs created in MatLab software (Mathworks Inc., Natick, MA, USA).

Co-activation was calculated according to Kellis ${ }^{19}$ : (antagonist activity / agonist activity + antagonist activity) * 100. This method has been used in studies that address coactivation, using the same absolute load before and after the intervention, and the signal measured with electromyography.

\subsection{Statistical analysis}

Statistical analysis was performed using SPSS software. The normality and homogeneity of all data (pre and post) were verified using the Shapiro Wilk and Levene tests, respectively. As the data presented normal distribution, for the comparison between the pre and post-intervention the paired Student $t$ test was performed, considering a level of significance of 0.05 .

\section{Results and Discussion}

13 individuals attended the study with mean $60.2 \pm 6.2$ years of age, body mass of $73.4 \pm 12.3 \mathrm{~kg}, 1.56 \pm 0.07 \mathrm{~m}$ of stature, and body mass index $30.1 \pm 5.2 \mathrm{~kg} / \mathrm{m} 2$. Firstly, the analysis indicated significant improvements $(P<0.05)$ after the 12-week combined training in the strength test in upper limbs (UL) (Figure 1A), elbow flexion and extension (EFE) (Figure 1C), strength test in lower limbs (LL test weight) (Figure 1D), sit-to-stand (SAS repetitions) (Figure 1F), and 6-minute walk test (Figure 1G). There was no statistical difference in the number of repetitions in the strength tests (Figures 1B and 1E).

Figure 1 - Motor skills tests pre- and post-exercise training $(n=13)$
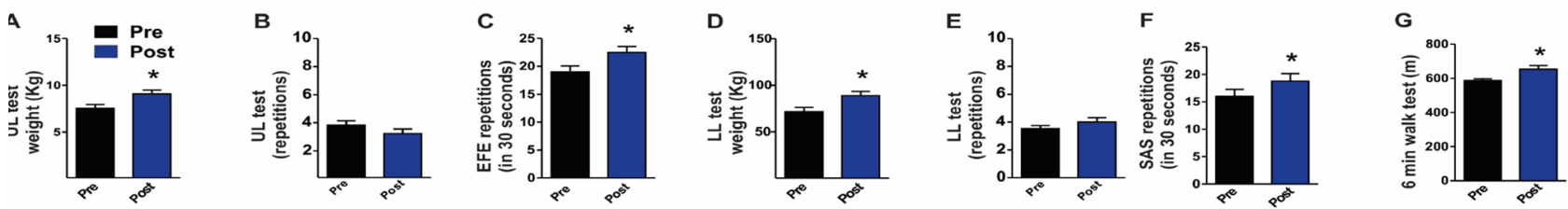

Note: (A) strength test in upper limbs (UL, $\mathrm{kg}$ ); (B) repetitions performed in the upper limbs dynamic load test; (C) elbow flexion and extension (EFE, repetitions); (D) strength test in the lower limbs (LL, kg); (E) repetitions performed in the lower limbs dynamic load test; (F) sit-to-stand (SAS, repetitions); (G) Six-minute walk test (meter). *p $<0.05$ (t-test).

Source: Research data.

There was a decrease in muscle activation post-intervention compared to pre-intervention, showing significant differences for the test of maximum dynamic load in the leg press equipment in the vastus medialis, semitendinosus, and biceps femoris muscles. No difference was observed in the decreased muscle activation in the sit-to-stand test (Table 1).

Table 1 - Mean \pm standard deviation of the electromyographic data in the tests of maximum dynamic load (LL) using the same absolute load before and after the intervention and of siting and standing $(\mathrm{SAS})(\mathrm{n}=13)$

\begin{tabular}{|c|c|c|c|c|}
\hline & \multicolumn{2}{|c|}{ LL (\%) } & \multicolumn{2}{|c|}{ SAS (\%) } \\
\hline & PRE & POST & PRE & POST \\
\hline VAL & $0.92 \pm 0.80$ & $0.87 \pm 0.10$ & $0.92 \pm 0.18$ & $0.92 \pm 0.34$ \\
\hline VAM & $0.93 \pm 0.11$ & $0.77 \pm 0.15^{*}$ & $1.10 \pm 0.51$ & $0.91 \pm 0.33$ \\
\hline REF & $0.82 \pm 0.15$ & $0.74 \pm 0.13$ & $1.13 \pm 0.51$ & $0.80 \pm 0.19$ \\
\hline SEM & $0.89 \pm 0.10$ & $0.75 \pm 0.13 *$ & $1.07 \pm 0.59$ & $0.83 \pm 0.39$ \\
\hline $\mathrm{BIF}$ & $0.86 \pm 0.11$ & $0.76 \pm 0.07 *$ & $1.06 \pm 0.39$ & $0.85 \pm 0.23$ \\
\hline $\begin{array}{l}\text { ote - } \\
\text { stus }\end{array}$ & $\begin{array}{l}\text { lower limb } \\
\text { eral; VAM: } \\
\text { losus; BIF: } \\
\text { contraction; }\end{array}$ & $\begin{array}{l}\text { leg press } 180 \\
\text { astus medial; }\end{array}$ & $\begin{array}{l}\text {; SAS: sit-to- } \\
\text { REF: rectus }\end{array}$ & $\begin{array}{l}\text { tand test; VAL } \\
\text { femoris; SEM }\end{array}$ \\
\hline
\end{tabular}

No decrease in the muscle activation was demonstrated for the exercise of maximum dynamic load in the unilateral biceps curl, or for the elbow flexion and extension test through electromyography in the upper limbs (Table 2).

Table 2 - Mean \pm standard deviation of electromyographic data in the tests of maximum dynamic load (UL) using the same absolute load before and after intervention and elbow flexion and extension (EFE) $(n=13)$

\begin{tabular}{|c|c|c|c|c|}
\hline & \multicolumn{2}{|c|}{ UL (\%) } & \multicolumn{2}{c|}{ EFE (\%) } \\
\hline & PRE & POST & PRE & POST \\
\hline BIB & $0.83 \pm 0.13$ & $0.76 \pm 0.86$ & $0.69 \pm 0.35$ & $0.69 \pm 0.17$ \\
\hline TRB & $0.78 \pm 0.15$ & $0.78 \pm 0.10$ & $0.72 \pm 0.40$ & $0.83 \pm 0.11$ \\
\hline
\end{tabular}

Note - UL: upper limbs (unilateral dumbbell thread, seated position); EFE: elbow flexion and extension; BIB: biceps brachii; TRB: triceps brachii. Data normalized by maximum voluntary contraction; * $P<0.05$ for pre x post comparison (Student's $t$ test)

Source: Research data.

In addition, the co-activation in the upper and lower limbs was analyzed, but no significance was found for any of the tests performed (Figure 2). 
Figure 2 - Co-activation of lower limbs and upper limbs pre- and post-exercise training $(n=13)$.
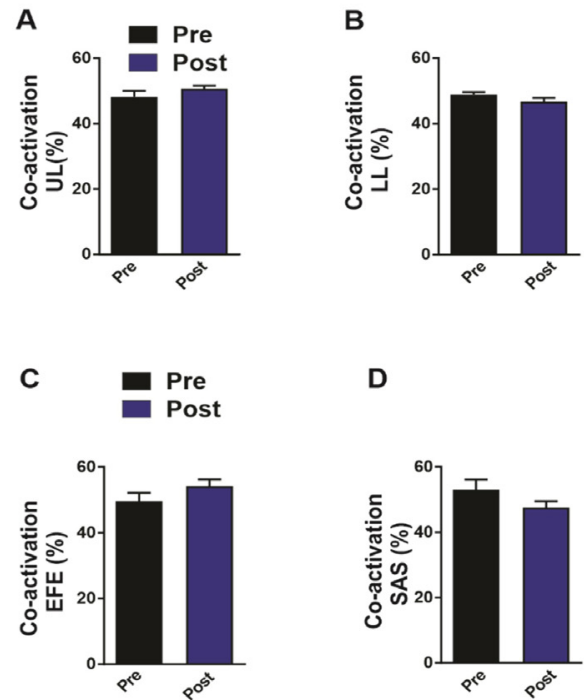

D

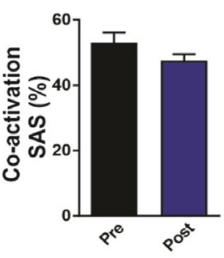

Note: (A) upper limbs muscle co-activation for maximal dynamic load (UL, \%); (B) lower limb muscle co-activation for maximal dynamic load (LL, \%); (C) muscular co-activation of the elbow flexion and extension (EFE, \%); (D) muscular co-activation of the sit-to-stand (SAS, \%). *p $<0.05$ (t-test).

Source: Research data.

The study focus was to analyze the effect of combined training on muscle function, strength, muscle activation, and co-activation in older women. It was demonstrated that the combined training protocol was effective, at least in part, for improving functional capacity and inducing reduced muscle activation in the older women's lower limbs.

Cadore $^{20}$ analyzed two training orders, utilizing 26 older adults divided into two groups: one group initially performed aerobic training followed by resistance training and the other group trained in the inverse order. In the study conclusion, the authors showed that the training order influenced strength capacity, being better in the group that started the session with strength training. The results herein on the strength levels in the maximum dynamic load tests in lower and upper limbs corroborate with the literature. Combined training focuses on the two main physical capacities, cardiorespiratory fitness, and muscle strength, which are independent predictors of mortality and morbidity ${ }^{20,21}$. In addition, this training program is recommended for middle-aged individuals ${ }^{20}$.

The intervention through combined training presented relevant results in strength in maximum dynamic load and upper and lower limb tests, since the participants presented increases in maximum absolute estimated load compared to the initial tests. Some studies have concluded that the interference effects can be minimized or even not occur when training is periodized and volume and intensity are controlled ${ }^{22,23}$. In line with this, Cadore ${ }^{24}$ did not find interferences in the training phenomena in the older population, demonstrating improvements in both neuromuscular and cardiorespiratory functions.
It is known that physical exercise offers several benefits during the aging process. However, few studies show the benefits of combined training on the older women's functional capacity. Herein, significant results were exhibited in the elbow flexion and extension and sit-to-stand tests, which assess the participants' functional strength ${ }^{20}$. In addition, as expected, the participants presented improvements in the pre- and post-intervention comparison through the 6-minute walk test, since combined training, when compared to aerobic training, does not present interference between the capacities $^{25}$; when combined training is compared to aerobic training, the gains are similar ${ }^{26}$. This study corroborates with what is presented in the literature.

Sillanpa ${ }^{27}$ assessed 63 sedentary men aged 40 to 65 years during 21-week physical training period, dividing them into three groups (aerobic training, strength training, and combined training) and demonstrating that combined training was effective in improving cardiorespiratory and muscular fitness. Studies have also shown that strength training promotes significant increases in muscle strength levels in adults and older adults, even with short intervention periods ${ }^{28}$. The current study also used a short intervention period (12 weeks), in physically active women. Considering the principle of trainability, physically inactive individuals present adaptations in the first months of training ${ }^{28}$, and, thus, it is believed that the positive results found in physically active women could be stronger in physically inactive individuals.

Increases in strength levels are predominantly related to neural adaptation, with the recruitment of more motor units, greater muscle activation, and decreased co-activation of antagonistic muscles in response to the physical training adaptation $^{29}$.

This study does not corroborate the literature since its results do not demonstrate a significant change in muscular co-activation after exercise training. However, as aforementioned, this is the first study to analyze muscle coactivation in a combined training program. Also, the amount of stimulation may not have been sufficient. Literature shows that eight weeks or more of strength training is sufficient to provide differences in muscle co-activation ${ }^{30}$.

On the other hand, Conlon and collaborators have demonstrated significant reductions in the antagonists muscular activation of the vastus medial in the sit-to-stand, providing a reduction in the stimuli through the association of strength gains and neural adaptations ${ }^{31}$. In the analysis herein, no statistical differences were found in the elbow flexion and extension test through electromyographic signals, or in muscle co-activation in the upper limb strength test. A possible hypothesis to justify these negative results may be the reduced number of exercises that directed stimuli to the biceps brachii and triceps brachii muscles associated with the sample of physically active women.

Sedentary older people present activation of contractions that occur simultaneously or commonly, increasing co- 
contraction and the risk of falls, however, in older trained individuals, body adjustments occur in a coordinated manner, reducing energy expenditure ${ }^{32}$. Adifference was observed in the activation of contractions in the dynamic maximum load test by the leg press device in the study participants, which although not sufficient to generate significant results for lower limb co-activation, indicates improved efficiency. In addition, the analysis in this study showed that a higher number of exercises were performed for the upper limbs than the lower limbs, which could justify the difference in strength gain in the limbs and their activation responses in this study.

The strength of the investigation herein was to analyze for the first time, in a well-controlled study, muscular coactivation in older women undergoing a combined training program. However, limitations in this study are recognized, such as sample size, 12-week intervention period (it is not known if a longer exercise program might result in effects on muscular co-activation), and the fact that $35 \%$ of the participants were not included in the analysis because they missed at least $25 \%$ of the training sessions or evaluations.

\section{Conclusion}

The exercise training protocol was partially effective for physically active older women, since the participants presented increases in absolute load post versus pre-intervention in the maximum dynamic load tests, in the number of repetitions in elbow flexion and extension, and in the sit-to-stand tests, as well as improved cardiorespiratory fitness. In addition, the protocol adopted for the combined training was able to facilitate alterations in the muscular activation of the lower limbs in physically active older women but it was not enough to improve the muscular co-activation. Studies with a training period longer than 12 weeks are necessary to investigate the real possibility of improvement in co-activation.

\section{Acknowledgments}

This study was financed in part by Coordenação de Aperfeiçoamento de Pessoal de Nível Superior - Brasil (CAPES) - Finance Code 001 - (grant 88882.180033/2018$01)$.

\section{References}

1. Organização Mundial de Saúde. Relatório mundial sobre envelhecimento e saúde: resumo. 2015. [acesso em 20 nov 2020] Disponível em http://apps.who.int/iris/ bitstream/10665/186468/6/WHO_FWC_ALC_15.01_por. pdf.

2. Niccoli T, Partridge L. Ageing as a risk fator for disease. Curr Biol 2012;22(17):R741-2. doi: https://doi.org/10.1016/j. cub.2012.07.024

3. Bouaziz W, Vogel T, Schmitt E, Kaltenbach G, Geny B, Lang PO. Health benefits of aerobic training programs in adults aged 70 years and over: a systematic review. Arch Gerontol Geriatr 2017;69(4):110-7. doi: https://doi.org/10.1016/j. lpm.2017.05.028
4. Mitchell WK, Williams J, Atherton P, Larvin M, Lund J, Narici M. Sarcopenia, dynapenia, and the impact of advancing age on human skeletal muscle size and strength; a quantitative review. Front Physiol 2012;3(2):777-80. doi: https://doi.org/10.3389/fphys.2012.00260

5. Faulkner JA, Larkin LM, Clafin DR, Brooks SV. Age related changes in the structure and function of skeletal muscles. Clin Exp Pharmacol Physiol 2007;34(2):1091-6. doi: https://doi. org/10.1111/j.1440-1681.2007.04752.x

6. Lopera VE, Arroyo P, Gutiérrez-Robledo LM, PérezZepeda MU, Cesari M. Mortality as na adverse outcome of sarcopenia. J Nutr Health Aging 2013;17(4):259-2. doi: https://doi.org/10.1007/s12603-012-0434-0

7. Pollock RD, O'Brien K, Daniels LJ, Nielsen KB, Rowlerson A, Duggal RA, et al. Properties of the vastus lateralis muscle in relation to age and physiological function in máster cyclists aged 55-79 years. Aging Cell 2018;17(4):12735. doi: https:// doi.org/10.1111/acel.12735

8. Cadore EL, Izquierdo M, Alberton CL, Pinto RS, Conceição $\mathrm{M}$, Cunha $\mathrm{G}$, et al. Strength prior to endurance intra-session exercise sequence optimizes neuromuscularand cardiovascular gains in elderly men. Exp Gerontol 2012;47(2):164-9. doi: https://doi.org/10.1016/j.exger.2011.11.013

9. Wilhelm EN, Rech A, Minozzo F, Botton CE, Radaelli R, Teixeira BC, et al. Concurrent strength and endurance training exercise sequence does not affect neuromuscular adaptations in older men. Exp Gerontol 2014;60(5):207-4. doi: https:// doi.org/10.1016/j.exger.2014.11.007

10. Robbins DW, Young WB, Behm DG, Payne WR. The effect of a complex agonist and antagonist resistance training protocol on volume load, power output, electromyographic responses, and efficiency. J Strength Cond Res 2020;24(4):1782-9. doi: https://doi.org/10.1519/JSC.0b013e3181dc3a53

11. Benedetti TRB, Antunes PC, Añez CRR, Mazo Z, Petroski ÉL. Reprodutibilidade e validade do Questionário Internacional de Atividade Física (IPAQ) em homens idosos. Rev Bras Med Esporte 2007;13(1):11-6. doi: 10.1590/ S1517-86922007000100004

12. Kraemer WJ, Ratamess NA, Fry AC, French DN, Maud PJ, Foster C. Strength training: development and evaluation of methodology. Science Op 1995;9(2):15-8.

13. Rikli RE, Jones CJ. Development and validation of a functional fitness test for community-residing older adults. Gerontologist 1999;7(2):129-1. doi: https://doi.org/10.1093/ geront/gns071

14. Rhea MR., Ball SD, Phillips WT, Burkett LNA. Comparison of linear and daily undulating periodized programs with equated volume and intensity for strength. J Strength Cond Res 2002;16(2):250-5. doi: https://doi.org/10.1519/15334287(2002)016<0250:acolad $>2.0$.co;2

15. Fleck SJ. Non-linear periodization for general fitness \& athletes. J Hum Kinet 2011;29(2):41-5. doi: https://doi. org/10.2478/v10078-011-0057-2

16. Tjønna AE, Lee SJ, Rognmo Ø, Stølen TO, Bye A, Haram $\mathrm{PM}$, et al. Aerobic interval training versus continuous moderate exercise as a treatment for the metabolic syndrome: a pilot study. Circulation 200822;118(4):346-54. doi: https:// doi.org/10.1161/CIRCULATIONAHA.108.772822.

17. Hermens HJ, Freriks B, Disselhorst-Klug C, Rau G. Development of recommendations for semg sensors and sensor placement procedures. J Electromyogr Kinesiol 2000;10(5):361-4. doi: https://doi.org/10.1016/s1050- 


\section{1(00)00027-4}

18. Burden A, Bartlett R. Normalisation of emg amplitude: an evaluation and comparison of old and new methods. Med Eng Phys 1999;21(4):247-7. doi: https://doi.org/10.1016/s13504533(99)00054-5

19. Kellis E, Arabatzi F, Papadopoulos C. Muscle co-activation around the knee in drop jumping using the co-contraction index. J Electromyogr Kinesiol 2003;13(3):229-8. doi: https://doi.org/10.1016/s1050-6411(03)00020-8

20. Cadore EL, Izquierdo M, Santos MG, Martins JB, Lhullier FLR, Pinto RS, et al. Hormonal responses to concurrent strength and endurance training with different exercise orders. J Strength Cond Res 2012;26(12):3281-8. doi: https:// doi.org/10.1519/JSC.0b013e318248ab26.

21. Cress ME, Buchner DM, Prohaska T, Rimmer J, Brown M, Macera $\mathrm{C}$, et al. Best practices for physical activity programs and behavior counseling in older adult populations. J Aging Phys Act 2005;13(1):61-4. doi: https://doi.org/10.1123/ japa.13.1.61.

22. Sahin UK, Kirdi N, Bozoglu E, Meric A, Buyukturan A, Ozturk A, et al. Effect of low-intensity versus high-intensity resistance training on the functioning of the institutionalized frail elderly. Int J Rehabil Res 2018;41(3):211-7. doi: https:// doi.org/10.1097/MRR.0000000000000285.

23. Valentini F, Perrone D, Stabile S, Pezzi O, Servidio S, De Marco R, et al. Differential kinetic dynamics and heating of ions in the turbulent solar wind. New J Phys 2016;18(12):125001. doi: https://doi.org/10.1088/13672630/18/12/125001.

24. Cadore EL, Izquierdo M. How to simultaneously optimize muscle strength, power, functional capacity, and cardiovascular gains in the elderly. Age (Dordr) 2013;35(6):2329-4. doi: https://doi.org/10.1007/s11357-012-9503-x.

25. Alcazar J, Losa-Reyna J, Rodriguez-Lopez C, Cruz RN, Alfaro-Acha A, Ara I, et al. Effects of concurrent exercise training on muscle dysfunction and systemic oxidative stress in older people with copd. Scand J Med Sci Sports 2019;29(10):1591-3. doi: https://doi.org/10.1111/sms.13494

26. Sakugawa RL, Moura BM, Orssatto LBDR, Bezerra ES, Cadore EL, Diefenthaeler F. Effects of resistance training, detraining, and retraining on strength and functional capacity in elderly. Aging Clin Exp Res 2019;31(1):31-9. doi: https:// doi.org/10.1007/s40520-018-0970-5.

27. Sillanpä E, Häkkinen A, Nyman K, Mattila M, Cheng S, Karavirta L, et al. Body composition and fitness during strength and/or endurance training in older men. Med Sci Sports Exerc 2008;40(5):950-8. doi: https://doi.org/10.1249/ MSS.0b013e318165c854.

28. Edholm P, Strandberg E, Kadi F. Lower limb explosive strength capacity in elderly women: effects of resistance training and healthy diet. J Appl Physiol (1985) 2017;123(1):190-6. doi: https://doi.org/10.1152/japplphysiol.00924.2016

29. Häkkinen, K Alen M, Kallinen M, Newton RU, Kraemer WJ. Neuromuscular adaptation during prolonged strength training, detraining and re-strength-training in middle-aged and elderly people. Eur J Appl Physiol 2000;83(1):51-2. doi: https://doi.org/10.1007/s004210000248.

30. Janusevicius D, Snieckus A, Skurvydas A, Silinskas v, Trinkunas E, Cadefau JA, et al. Effects of high velocity elastic band versus heavy resistance training on hamstring strength, activation, and sprint running performance. J Sports Sci Med 2017;16(2):239-6.

31. Conlon JA, Newton RU, Tufano JJ, Peñailillo LE, Banyard HG, Hopper AJ, et al. The efficacy of periodised resistance training on neuromuscular adaptation in older adults. Eur J Appl Physiol 2017;117(6):1181-4. doi: https://doi. org/10.1007/s00421-017-3605-1.

32. Santos SM, da Silva RA, Terra MB, Almeida IA, de Melo $\mathrm{LB}$, Ferraz HB. Balance versus resistance training on postural control in patients with parkinson's disease: a randomized controlled trial. Eur J Phys Rehabil Med 2017;53(2):173-3. doi: https://doi.org/10.23736/S1973-9087.16.04313-6. 